\title{
Uso del smartphone en el periodismo actual: ¿hacia un nuevo modelo mediático?estudio de caso del contexto malagueño
}

\section{Uses of the smartphone in current journalism. towards a new media model? case study of the context of malaga}

\author{
Marta Gil Ramírez \\ Universidad de Málaga \\ martagr@uma.es \\ ORCID: 0000-0002-0577-5628 \\ Ruth Gómez de Travesedo Rojas \\ Universidad de Málaga \\ ruthgtr@uma.es \\ ORCID: $\underline{0000-0003-4452-5928}$
}

\section{RESUMEN}

En los últimos años, el avance de la tecnología está introduciendo transformaciones significativas en el sistema mediático tradicional. La penetración de los smartphones y el uso masivo de los mismos para el consumo informativo obliga a los medios de comunicación a actualizarse y adaptarse a la nueva realidad social. Ubicuidad, inmediatez e interactividad son, hoy en día, las características predominantes de la producción, difusión y consumo de contenidos mediáticos. Las noticias móviles están ganando una importancia crucial para los medios tradicionales (Westlund, 2013) y los profesionales de la información deben afrontar el ajuste de sus rutinas productivas a este nuevo escenario. La presente investigación, tomando como estudio de caso el entorno mediático malagueño, se propone conocer la 
forma en la que los periodistas, así como las empresas de medios, se adaptan al uso de la denominada "cuarta pantalla" (Aguado y Martínez, 2008; Miller, 2014) de los Smartphone o teléfonos inteligentes, unos dispositivos considerados ya como el "metamedio del siglo XXI” (Márquez, 2017).

Para ello se emplea la entrevista en profundidad como metodología cualitativa-descriptiva junto a la observación participante, dada la condición de redactora, en los servicios informativos de PTV Málaga, de una de las autoras del texto.

Los resultados evidencian la polivalencia (González, 2013) como un factor prominente entre los periodistas locales que, a diario, emplean el Smartphone en sus rutinas productivas. La investigación concluye que, en el ámbito local, aunque con excepciones, las empresas mediáticas tienden a aprovechar las potencialidades que actualmente ofrecen los teléfonos inteligentes.

\section{ABSTRACT}

In recent years, the advancement of technology is introducing significant transformations in the traditional media system. The penetration of Smartphones, and the massive use of them for informative consumption, forces the media to update and adapt to the new social reality. Ubiquity, immediacy and interactivity are, nowadays, the predominant characteristics of the production, diffusion and consumption of media content. Mobile news is gaining crucial importance for traditional media (Westlund, 2013) and information professionals must confront the adjustment of their productive routines to this new scenario.

The present investigation, taking as a case study the media environment of Malaga, proposes to know the way in which journalists, as well as media companies, adapt to the use of the so-called "fourth screen" (Aguado and Martínez, 2008; Miller, 2014) of smartphones, some devices already considered the "metamedia of the 21 st century" (Márquez, 2017).

For this, the in-depth interview is used as a qualitative-descriptive methodology together with the participant observation, given the condition of editor, in the information services of PTV Málaga, by one of the authors of the text.

The results show the versatility (González, 2013) as a prominent factor among local journalists, who daily use Smartphones in their productive routines. The research concludes that, at the local level, although with exceptions, media companies tend to take advantage of the potentials that smartphones currentlyoffer. 


\section{PALABRAS CLAVE | KEYWORDS}

Periodismo,medios de comunicación, teléfono inteligente, teléfono móvil, multimedia. Journalism, media, smartphone, mobile phones, multimedia.

\section{INTRODUCCIÓN}

Hace poco más de veinte años el consumo de información a través de los medios tradicionales escasamente se parecía al actual. Un consumo tipo podría representarse en una persona que leía el periódico a primera hora, veía el telediario en su edición del medio día o de la noche y escuchaba algún boletín radiofónico a lo largo del día. Tras la llegada de Internet, los medios masivos no tardaron en tener su versión digital en la Red. En este contexto, cualquiera en un PC podía consultar la información que deseara cuando quisiera, el consumo se liberaba así de restricciones temporales, pero aún quedaba la limitación espacial. Hoy en día, Smartphone en mano, el consumo es ubicuo.La información se puede consultar everywhere, at all times. "El móvil representa en la actualidad un paradigma de la concepción MacLuhaniana a partir de la cual una tecnología se concibe como una extensión del cuerpo humano, de nosotros mismos" (López, Westlund y Silva, 2015, p. 129). Con los primeros teléfonos móviles, el consumo de información se benefició de la portabilidad y la ubicuidad. Con el siguiente escalón, esto es, con la llegada de los smartphones, a estas características se suman otras propias de los dispositivos multifunción, "como la multimedialidad, la hipertextualidad y la interactividad facilitada por los altos niveles de usabilidad" (Canavilhas, 2013, p. 25).

"Los continuos avances en telefonía móvil están haciendo de este dispositivo la principal tecnología de la con- vergencia, una especie de 'mando a distancia universal' capaz de almacenar los diferentes usos y prestaciones de los múltiples sistemas mediáticos" (Márquez, 2017, p. 61).

La llegada de Internet obligó a los medios a dar el salto de lo analógico a lo digital. La transformación digital no solo supuso la ampliación y la creación de nuevos medios, sino que además alimentó nuevas formas de contar historias, renovadas modalidades expresivas y complejos sistemas de interacción comunicativa (López et al., 2015).

Así, la implantación de los teléfonos inteligentes empuja a las empresas mediáticas hacia un nuevo modelo difícil de titular. Para Márquez (2017, p. 62) "hoy en día los medios ya no son simplemente medios sino postmedios, hipermedios, multimedios, o metamedios, que entran a su vez en complejas redes transmediáticas que los llevan de un lugar a otro, de un soporte a otro". Siguiendo a este autor, el Smartphone representaría el nuevo paradigma de metamedio en el siglo XXI.

El teléfono móvil (en su versión Smartphone) puede considerarse como un nuevo tipo de `metamedio', esto es, una plataforma que alberga muchos medios existentes y nuevos, que incluye la mayoría de las tecnologías y técnicas de los medios existentes así como la invención de otras nuevas. 
(Márquez, 2017, p. 61)

Estaríamos, por tanto, "inmersos en un nuevo contexto comunicativo que bien pudiéramos bautizar como la era de los smartphones" (Costa, 2012, p. 244).

Muchos estudiosos se refieren a este dispositivo como "la tercera" (Kerckhove, 2005) o "la cuarta pantalla" (Aguado y Martínez, 2008; Miller, 2014), por detrás de los históricos medios tradicionales como la televisión, el cine e Internet (Ahonen, 2008). Otros (Gardner y Davis, 2014) la sitúan ya como "la primera pantalla" tratando de enfatizar la importancia y la naturalidad que el Smartphone adquiere para las nuevas generaciones, nativos de la telefonía móvil, como herramienta de consulta e interacción con el mundo que les rodea. En este contexto, "medios tradicionales (de todos los sectores, prensa, radio y televisión), así como medios digitales intentan dotar de contenidos a las nuevas pantallas" (Costa, 2012, p. 244).

La eclosión de las redes sociales, ahora disponibles en cualquier momento y lugar a través del Smartphone, está transformando por completo el tradicional sistema mediático. El Smartphone se ha convertido en "un canal de comunicación directa entre los medios de comunicación y los usuarios" (Canavilhas, 2013, p. 25). La sociedad civil cuenta así con la herramienta que posibilita su participación activa, también en los contenidos e informaciones de los medios de comunicación de masas, alterando las tradicionales relaciones de poder, donde la esfera pública solía ser diana de la esfera mediática (y de la esfera política). Esta tecnología ha hecho tambalear el monopolio de la información que, hasta hace poco, ostentaban los medios de comunicación tradicionales. El periodista ya no controla la agenda, ni elige los con- tenidos, ni jerarquiza la información (Reigosa, 2007). Las tecnologías actuales han logrado convulsionar profundamente las bases, relaciones y equilibrios sobre los que se asentaba la creación-producción y la distribución de la comunicación en la anterior etapa (Bustamante, 2011). El periodismo se ve abocado, así, a una alteración y redefinición de las relaciones de poder y a una disolución de las líneas divisorias existentes (Williams y DelliCarpini, 2011).Para López et al. (2015) la irrupción de los dispositivos móviles inteligentes ha abocado a las industrias de medios a la introducción de cambios radicales en los modos de producción y distribución de contenidos, con estrategias para la producción multiplataforma y para el aprovechamiento del potencial de los usuarios no solo como fuente, sino también como generadores de mensajes.

Los dispositivos móviles (teléfonos y tablets) se han convertido ya en el principal medio de acceso a internet en España. Este cambio profundo en la forma de acceder a la Red supone lógicamente un cambio en la forma en que el público se informa e interactúa con los contenidos informativos. (Varona, Sánchez y Arrocha, 2017, p. 641)

"El Smartphone (...) ha creado nuevos medios y aplicaciones que ofrecen nuevas posibilidades de acción e interacción mediática y reconfiguran el modo en que concebimos los demás medios, transformando nuestra relación con ellos" (Márquez, 2017, p. 68).

Así, los cambios que el Smartphone introduce en el sistema mediáticose podrían sintetizar alrededor de tres ejes según González (2013): 
a) Se modifica el acceso a las fuentes, ya que aparecen nuevos actores con voz propia y capaces de incidir en el discurso informativo: el propio público, ubicuo y activo. b) Se gana en rapidez y agilidad en las coberturas, que se pueden efectuar desde el mismo lugar en el que ocurren los hechos noticiosos incorporando a la vez la voz de la audiencia. c) Se transforman los contenidos, que se tienen que adaptar al nuevo entorno de comunicación móvil que implica un continuo diálogo con el usuario.(p.8)

Atendiendo a estos cambios, Sainz de Baranda, Limón y Herrero (2012) señalan que:

Los medios de comunicación ya han comenzado a desarrollar verdaderas salas de redacción multimedia desde las que se gestionan diferentes soportes con el objetivo de presentar la información, de múltiples maneras, a un público que la consumirá a través de diversos canales: contenidos diversos para audiencias móviles. (p.62)

El auge que actualmente experimenta el mercado de dispositivos móviles y su uso en el consumo mediático aconseja el abordaje académico de esta cuestión, máxime cuando la comunicación vive un momento convulso con los medios replanteándose su modelo de negocio (Casero-Ripollés, 2010).

\subsection{TRANSFORMACIONES QUE INTRODUCE EL SMARTPHONE EN LAS RUTINAS PERIODÍSTICAS}

El nuevo escenario de las Tecnologías de la Información y la Comunicación, entre las que posicionamos el Smartphone, supone, irrevocablemente, una remodelación de las funciones propias del trabajador en muchos ámbitos del mercado laboral. El periodismo no ha sido ajeno a las transformaciones que imponen los teléfonos inteligentes en sus rutinas productivas, máxime si atendemos a los resultados de la investigación realizada por Varona et al. (2017) en la que los autores concluyen que "los contenidos informativos elaborados por medios de comunicación son una de las cosas más demandadas y consultadas por los usuarios españoles de smartphones" (p. 645). "Una de las esferas en las que el impulso transformador de la tecnología digital se ha dejado sentir con más fuerza tiene que ver con el perfil profesional del periodista, más concretamente con sus competencias" (González, 2013, p.9).

Hace un par de décadas el periodista se caracterizaba por su conocimiento de los códigos mediáticos. Ya fuera en prensa, radio o televisión, con las especificidades de cada medio, la escritura periodística era la herramienta básica del profesional para transcribir, interpretar, trasladar a los lectores, oyentes o audiencia, la información. Hoy en día, el Smartphone impone a la profesión muchas otras tareas. "Los dispositivos móviles tipo smartphones y tablets vienen a añadir un elemento nuevo, el de la movilidad, con suficiente capacidad para transformar las competencias de los profesionales del periodismo" (González, 2013, p.8).

Características que posibilitan los teléfonos inteligentes como la ubicuidad, la multimedialidad, la hipertextualidad o la posibilidad de instantaneidad, suponen "cambios en los procesos de producción, distribución y consumo informativos que implican nuevas habilidades profesionales para a) gestionar las fuentes de información, b) elab- 
orar contenidos periodísticos y c) relacionarse con una audiencia cada vez más participativa" (González, 2013, p.7).

El periodista en la era de la convergencia (Jenkins, 2006) debe estar provisto de las habilidades necesarias para, en la cobertura de un evento, grabar un video, editarlo, realizar fotografías y escribir la crónica adaptándola posteriormente a las distintas plataformas en las que se vaya a publicar.

El periodista que trabaja en un entorno móvil se perfila como el profesional adecuado para confeccionar noticias concebidas a modo de titulares o de típico flash de agencia, con sólo la información básica combinada a veces con una fotografía o un pequeño video (...) Se trata aquí de construir un relato global a partir de tantos mensajes breves como novedades ocurran, acompañados de los recursos audiovisuales pertinentes (fotografías, vídeos o sonido). (González, 2013, p.14)

Hoy, el periodista puede actualizar su medio con textos o elementos gráficos como vídeos o imágenes desde el lugar de los hechos, a través de su dispositivo móvil y el usuario puede recibir las informaciones y consultarlas en su terminal, en cualquier sitio, al instante siguiente (Melle y López, 2017).

Márquez (2017, p. 66) nos habla de "un nuevo tipo de identidad para el periodista, el denominado periodista MOJO o Mobile Journalist, aquel que es capaz de montar una noticia desde la calle a partir de su Smartphone". Quinn (2009, p.10) también habla al respecto del periodista móvil como aquel "que usa principalmente el teléfono móvil como mecanismo para recopilar y distribuir noticias, ya sea en formato textual o audiovisual (sonido, imágenes fijas o en movimiento) o bien combinándolos". Diversos autores (Huang, Rademakers, Fayemiwo, y Dunlap,2004; García Avilés, Meier, Kaltenbrunner, Carvajal y Kraus, 2009; Micó, 2011; González, 2013) emplean el término "polivalente" para definir al periodista en el contexto actual. Así, un periodista polivalente sería aquel que produce noticias para dos o más medios usando las herramientas tecnológicas pertinentes para cada estadio del proceso (García Avilés et al., 2009); aquel que cuenta con más flexibilidad y habilidades técnicas para trabajar en múltiples plataformas, con diferentes lenguajes y sobre diversos temas (Huang et al., 2004); aquel que desarrolla su cometido principalmente a pie de calle, de manera individual y autónoma, con la suficiente habilidad como para suministrar datos de manera regular y continua, en formato textual y audiovisual e incorporando las aportaciones que al respecto pueda efectuar la audiencia (González, 2013) o aquel "que usa los dispositivos móviles como herramienta de trabajo" (Micó, 2011, p. 204). La polivalencia aludiría así a "la capacidad y habilidad que tiene el periodista de ejercer simultáneamente diversos cometidos de forma habitual" (González, 2013, p.9). "No hay duda de que el desarrollo de la tecnología ha sido básico para el avance de estos nuevos perfiles de informadores" (Melle y López, 2017, p. 150). Esta realidad se hace aún más patente en las empresas mediáticas de ámbito local, donde en muchas ocasiones y como recuerda González (2013, p. 11) "la polivalencia no se concibe como una opción sino como una obligación".

Sobre las potencialidades que traen consigo los smartphones para el quehacer periodístico, bien podríamos cuestionarnos: ¿suponen las múltiples posibilidades de estos dispositivos una ventaja o un inconveniente en el 
trabajo periodístico? La mayoría pensaría que los avances tecnológicos son siempre positivos, aunque entre los miembros del gremio también existen voces que apuntan a los perjuicios que genera el mayor volumen de trabajo derivado de las nuevas funciones que le aporta al periodismo el Smartphone, lo que deviene en el deterioro de la calidad de la profesión y de la producción de contenidos. Aunque sumamente interesante, no es este el debate que nos ocupa, máxime cuando, para bien o para mal, la presencia de los teléfonos inteligentes en las rutinas periodísticas es hoy una realidad. Centrémonos, por tanto, no en juzgar los cambios, sino en conocerlos y tratar de sintetizarlos de manera científica.

Parece cierto que los dispositivos multifunción han transformado la producción y distribución de contenido mediático y el consumo de información periodística. La reordenación de las rutinas obliga al periodista a "enfrentarse a los desafíos de la fase de la sociedad en red que camina bajo la alargada sombra de los dispositivos móviles" (López et al., 2015, p. 132). Hoy en día el periodismo compite por la primacía de la información mediante la instantaneidad y la adaptación de los contenidos a las distintas plataformas digitales, entre ellas las Apps para dispositivos móviles y las redes sociales. "El usuario (...) ya no va a la búsqueda de información, sino que la información va en busca del usuario y lo encuentra en un punto concreto de su itinerario" (Márquez, 2017, p. 67). 'La información llega 'en movimiento' y su influencia está cambiando la experiencia de los consumidores de información” (Varona et al., 2017, p. 642). Así, los ciudadanos, hoy en día, "buscan las noticias ya no en el quiosco ni en la televisión o en sus ordenadores personales, sino que persiguen la rapidez y la comodidad que supone sacarlas de sus bolsillos" (López et al., 2015, p. 138). Los usuarios tienen a su disposición toda la información a un solo clic o pulsación táctil y en este mercado de sobreexposición mediática, al periodista ya no le basta con una buena redacción, sino que tendrá que adaptarse a las destrezas tecnológicas que le imponen las nuevas herramientas digitales, si pretende sobrevivir en un mercado cada vez más saturado y competitivo.

Así, "como receptor o como herramienta para los periodistas, el Smartphone introduce cambios importantes en la actividad periodística, obligando a una reflexión sobre el tema" (Canavilhas, 2013, p.22). "Aunque su uso como soporte informativo es aún tímido, su progresiva extensión en el mercado obliga a plantearse de qué manera cambia la manera de trabajar de los periodistas que lo usan” (González, 2013, p.16).

El consumo de información a través de los móviles, de acuerdo con autores como Westlund (2015) “está poco estudiado desde la academia, especialmente en España y es por ello un campo atractivo en términos científicos" (p. 152). "Es además un fenómeno muy complejo y lleno de facetas y enfoques diferentes que conviene ir delimitando" (Varona et al., 2017, p. 642).

Los cambios que introducen los smartphones en la rutina periodística abren la posibilidad a un amplio ámbito de estudio que aún carece de un recorrido que aborde y delimite la cuestión de manera científica. Así, tratando de reflejar los trabajos recientes que, de un u otro modo se relaciona con el fenómeno,los de Sánchez (2011), Sainz de Baranda et al. (2012) y González (2013) serían los más próximos a la presente investigación en cuanto a su objeto de estudio, esto es, analizar la repercusión que el Smartphone ha tenido y está teniendo en las rutinas productivas periodísticas. Quiróz (2016) desarrolla a nivel teórico cada uno de los recursos -grabación, edición, 
conexión, etc.- que ofrecen al periodismo los smartphones.Otros estudios, con planteamientos próximos al nuestro, analizan la adaptación de los medios de comunicación -televisión en el caso de Micó (2011), periódicos en el caso de López et al. (2015), Marta Lazo y García Idiakez (2014) y Said et al. (2013), medios nativos digitales en el caso de Costa (2012)- al tipo de periodismo que imponen los nuevos teléfonos inteligentes. La propuesta de Márquez (2017) supone una interesante reflexión teórica acerca del Smartphone como nuevo paradigma mediático.

\section{OBJETIVOS}

El objetivo general de esta investigación es conocer cuál es el modelo que están aplicando las empresas mediáticas malagueñas en el actual contexto 2.0 y cómo los profesionales de la información se adaptan al uso de lo que hoy en día se considera ya el "metamedio del siglo XXI" (Márquez, 2017), esto es, la denominada "cuarta pantalla" (Aguado y Martínez, 2008; Miller, 2014) de los Smartphone o teléfonos inteligentes.

Atendiendo a este objetivo general, respecto al modelo que aplican los medios de comunicación en Málaga en el actual contexto 2.0, los objetivos específicos son los siguientes:

- Conocer si las empresas mediáticas cuentan con App específica para la distribución de contenidos a través de dispositivos móviles.

- Constatar si los medios de comunicación examinados cuentan con personal o departamento específico encargado de adaptar y publicar los contenidos en los distintos soportes y plataformas de difusión (véanse web, redes sociales, App móvil, etc.)

- Certificar si las empresas mediáticas imparten formación a sus periodistas sobre el correcto uso de las herramientas que presentan los smartphones para el desarrollo de sus funciones.

Para profundizar en cómo los profesionales de la información se adaptan al uso del Smartphone en sus rutinas productivas, los objetivos específicosse estructuran en base a tres ejes:

a) El Smartphone como dispositivo multifunción

- Acreditar en qué forma y medida, el uso de los teléfonos inteligentes ha cambiado las rutinas productivas de los profesionales de la información.

- Conocer cuáles son las ventajas e inconvenientes que encuentra el sector en el empleo del Smartphone.

- Al margen de las tradicionales labores de recopilación de datos y redacción de la pieza informativa, registrar las nuevas funciones que los profesionales realizan tras la llegada de los teléfonos inteligentes al ámbito periodístico.

- Constatar en qué medida los periodistas se consideran dentro de la denominación "Mobile Journalist".

b) El Smartphone como "metamedio” (Márquez, 2017)

- Comprobar si los redactores entrevistados emplean su Smartphone como medio para infor- 
marse de la actualidad y a través de qué aplicaciones concretas lo hacen.

c) El Smartphone como fuente de información periodística este ámbito no se corresponden con el perfil que trata de examinarse en esta investigación. Con todo, la muestra queda constituida por periodistas de 11 medios de comunicación locales.
- Atestiguar el uso que el periodista hace de su Smartphone como fuente de información a través de las aportaciones de ciudadanos particulares o colectivos sociales y en qué medida se nutren de dichas aportaciones en la elaboración del contenido periodístico.

\section{Tabla 1. Universo muestra}

\begin{tabular}{|c|c|c|}
\hline TELEVISIONES & RADIOS & DIARIOS \\
\hline Onda Azul $^{2}$ & Onda Azul Radio $^{3}$ & Sur \\
\hline PTV Málaga & Onda Cero Málaga $^{4}$ & La Opinión de Málaga \\
\hline 101 TV & Ser Málaga $^{4}$ & Málaga Hoy \\
\hline & Cadena COPE Málaga & Viva Málaga \\
\hline
\end{tabular}

\section{METODOLOGÍA}

Este análisis exploratorio, asentado en el estudio de caso de los medios de comunicación en Málaga, emplea una metodología cualitativa-descriptiva basada en entrevistas en profundidad a periodistas de radios, diarios y televisiones de Málaga capital, así como en la observación participante, dada la condición, de una de las autoras, de redactora en los servicios informativos de PTV Málaga.

La muestra parte del registro oficial de los medios de comunicación en Málaga de la Junta de Andalucía ${ }^{1}$.De los medios que recoge dicho registro, se toman en consideración aquellas radios, diarios y televisiones con sede en Málaga capital (excluyendo así los medios del resto de municipios de la provincia) y aquellos que cuentan con al menos dos personas en la plantilla (descartando, por tanto, los medios de ámbito nacional o autonómico que cuentan, exclusivamente, con un corresponsal en la ciudad). En el caso de las radios se prescinde también de las radio-fórmulas musicales, dado que los profesionales de

\section{Fuente: elaboración propia}

Se da la peculiaridad de que la periodista que trabaja para el periódico Viva Málaga, lo hace al mismo tiempo para OndaluzTV, dado que ambos medios pertenecen al Grupo Online Publicaciones del Sur. Encontramos también en la muestra el caso inverso: pese a que Canal Málaga RTV cuenta con radio y televisión, el grupo no utiliza profesionales polivalentes a nivel mediático (Micó, 2006), sino que opta por la especialización periodística.

A los 11 profesionales de los distintos medios de comunicación se les realizó una entrevista en profundidad estructurada en tres ejes que tratan de ahondar en las características del uso del Smartphone como a) dispositivo multifunción, b) metamedio (Márquez, 2017), c) fuente de información periodística, así como en el modelo mediático que aplican sus empresas de comunicación.

Por otra parte, la condición de una de las autoras de redactora en los servicios informativos de PTV Málaga, le posibilita estar estrechamente familiarizada con 
los periodistas locales y con el contexto diario en el que estos desarrollan sus funciones, lo que a su vez le permite teorizar al respecto de los aspectos cualitativos del fenómeno a través de la observación participante.

\section{RESULTADOS}

“El mundo periodístico con el uso de los smartphones ha dado un giro de 180 grados. Con ellos, llevas la oficina encima” (Pilar R. Quirós, Diario SUR). Todos los entrevistados coinciden en la enorme transformación que ha supuesto el Smartphone en sus rutinas diarias. "El móvil ha modificado y cambiado todos nuestros hábitos a la hora de trabajar" (Manuel García, Canal Málaga RTV Televisión-). La posibilidad de la constante actualización y la mayor facilidad en el acceso a la información, pilares fundamentales en la profesión periodística, son dos de los cambios más aludidos. "A través del Smartphone estoy actualizada en cualquier momento, independientemente de mi ubicación a lo largo del día" (Romina Zace, 101 TV). "El Smartphone permite estar en contacto continuo con los distintos medios de comunicación e información. De hecho, lo más habitual es mirar el teléfono nada más levantarse, mientras que antes era necesario escuchar la radio o leer la prensa" (Manuel Álvarez, Onda Cero Radio). "Esta profesión te exige estar al tanto de la actualidad. Un dispositivo con conexión a internet es fundamental" (Andrés García, Cadena COPE Málaga). Del mismo modo, el carácter digital de los dispositivos multifunción ha hecho que las tradicionales herramientas periodísticas (bolígrafo, papel, grabadora) se actualicen. "Como herramienta unifica tres herramientas: cámara, grabadora y bloc de notas. En este sentido, diría que ha facilitado el trabajo" (Matías Stuber, La Opinión de Málaga). "El Smartphone nos ha ahorrado tiempo y
Apps como Whatsapp se ha 'oficializado' a la hora de cerrar temas como periodista" (Celia Bermejo Pérez, Canal Málaga RTV -Radio-).

Los periodistas interrogados consideran, casi por unanimidad, que el Smartphone "ha mejorado la recepción de información y la conexión con las fuentes" (Juan Miguel Domínguez, PTVTELECOM). Todos los entrevistados afirman utilizar su teléfono inteligente para mantenerse informado de las últimas noticias, empleando para ello, de forma mayoritaria, Twitter o las webs y/o aplicaciones de los principales medios de comunicación -especialmente prensa- locales y nacionales.

Con la llegada de los teléfonos inteligentes el profesional del periodismo ha visto modificadas sus funciones. Al margen de las tradicionales labores de recopilación de datos y redacción de la pieza informativa, el Smartphone ha abierto la puerta a una mayor polivalencia (González, 2013). El redactor actual, realiza fotografías (Ángel Recio, Málaga Hoy; Jesús Sánchez, Cadena SER Málaga; Pilar R. Quirón, Diario SUR), graba y edita vídeos (Romina Zace, 101 TV; Jesús Sánchez, Cadena SER Málaga), publica y comenta en redes sociales (Celia Bermejo, Canal Málaga RTV -Radio-; Ángel Recio, Málaga Hoy; Manuel García, Canal Málaga RTV -Televisión--; Jesús Sánchez, Cadena SER Málaga). "La capacidad de memoria y definición de los terminales te permite grabar vídeos y hacer fotos de buena calidad visual y esto es algo que pude solucionar la cobertura de algún hecho no programado" (Marta Jiménez, Viva Málaga/OndaluzTV). El Smartphone también ha posibilitado que entre las nuevas funciones del profesional de la información esté el envío de contenido actualizado a su empresa desde la ubicación de la noticia. "Lo utilizo cuando hay que mandar una información con cierta urgencia a los compañeros de la web” (Matías Stuber, La Opinión de Málaga). “A la hora 
de enviar el trabajo a la emisora ya no es necesario volver al centro de trabajo" (Manuel Álvarez, Onda Cero Radio). El periodista del siglo XXI incluso emite en directo desde su teléfono móvil. "Una aplicación me permite conectarme con la emisora y entrar desde el exterior en emisión con calidad aceptable" (Manuel Álvarez, Onda
Cero Radio). Pilar R. Quirón (Diario SUR) sintetiza el carácter ventajoso de esta multifuncionalidad "me ha hecho libre a la hora de montar mis informaciones".

Pese a la constatada polivalencia profesional y las nuevas funciones que, hoy en día, realizan los periodistas, sólo dos de los once entrevistados se consideran dentro de la denominación Mobile Journalist (Márquez, 2017; Quinn, 2009). “Antes solo usaba grabadora y libreta y ahora uso el teléfono como grabadora de voz, fotos, imágenes, libreta, montaje, envíos de correos y redes sociales para difundir noticias" (Jesús Sánchez, Cadena SER Málaga). "Con mi Smartphone grabo sonido, vídeo que edito para RRSS, fotos y material para noticias, reportajes, entrevistas... (Celia Bermejo Pérez, Canal Málaga RTV -Radio-). El hecho de que la mayoría de los redactores no se enmarquen dentro del denominado perfil de "periodista móvil" tiene que ver con que la mayoría sigue acudiendo a su lugar de trabajo para maquetar la información a través de su PC. "Edito mis informaciones en ordenador con teclado" (Pilar R. Quirós, Diario SUR). "Soy más de teclado de pc" (Marta Jiménez, Viva Málaga/OndaluzTV).

\section{Tabla 2. Adaptación de los periodistas al uso del Smartphone en sus rutinas productivas}

\begin{tabular}{|c|c|c|c|c|}
\hline \multirow{5}{*}{$\begin{array}{l}\text { Como dispositivo } \\
\text { multifunción }\end{array}$} & $\begin{array}{l}\text { Principales } \\
\text { cambios }\end{array}$ & \multicolumn{3}{|c|}{$\begin{array}{l}\square \text { Posibilidad constante actualización } \\
\square \text { Mayor facilidad en el acceso a la información } \\
\square \text { Actualización herramientas periodísticas }\end{array}$} \\
\hline & \multirow[b]{2}{*}{$\begin{array}{l}\text { Ventajas e } \\
\text { inconvenientes }\end{array}$} & \multirow[b]{2}{*}{ Inmediatez } & Ventajas & $\begin{array}{l}\square \text { Rapidez } \\
\square \text { Actualidad }\end{array}$ \\
\hline & & & Inconvenientes & $\begin{array}{l}\square \text { Desprofesionalización } \\
\square \text { “Hombre orquesta” } \\
\square \text { Guerra entre periodistas } \\
\square \text { Reducción de costes } \\
\square \text { Errores en contenidos }\end{array}$ \\
\hline & Nuevas funciones & \multicolumn{3}{|c|}{$\begin{array}{l}\square \text { Realizar fotografías } \\
\square \text { Grabar y editar vídeos } \\
\square \text { Publicar y comentar en redes sociales } \\
\square \text { Enviar contenido actualizado desde la ubicación de la } \\
\text { noticia } \\
\square \text { Emitir en directo desde su teléfono móvil }\end{array}$} \\
\hline & $\begin{array}{l}\text { Consideración } \\
\text { MOJO }\end{array}$ & \multicolumn{3}{|c|}{$\begin{array}{l}\text { Sólo dos de los once entrevistados se consideran dentro de } \\
\text { la denominación Mobile Journalist }\end{array}$} \\
\hline $\begin{array}{l}\text { Como } \\
\text { "metamedio" }\end{array}$ & \multicolumn{4}{|c|}{$\begin{array}{l}\text { Todos los periodistas usan su teléfono inteligente para mantenerse informado de } \\
\text { las últimas noticias } \\
\text { (A través de: Twitter o las webs y/o aplicaciones de los principales medios de } \\
\text { comunicación) }\end{array}$} \\
\hline $\begin{array}{l}\text { Como fuente } \\
\text { de información } \\
\text { periodistica }\end{array}$ & \multicolumn{4}{|c|}{$\begin{array}{l}\text { Nivel bajo debido a la necesidad de contrastar la veracidad de la información } \\
\text { recibida y de la fuente }\end{array}$} \\
\hline
\end{tabular}

Fuente: elaboración propia a partir de los datos obtenidos a través de las entrevistas en profundidad 
1Todas las empresas mediáticas a las que pertenecen los entrevistados, a excepción de dos, cuentan con una App específica para la distribución de contenidos a través de dispositivos móviles. La mayoría de los medios de comunicación en Málaga no imparten formación a sus periodistas para el aprovechamiento, en sus rutinas productivas, de las herramientas que presentan los smartphones. Sí lo hacen Cadena Cope Málaga, Cadena Ser Málaga y Diario SUR.

Málaga Hoy o Canal Málaga RTV han impartido algún curso de este tipo a su personal de manera casi residual. "Una vez nos dieron un curso de un par de horas sobre la nueva web y cómo acceder para meter contenido desde el or-

Tabla 3. Gestión de la información en función del soporte en el que se difunda

\begin{tabular}{|c|c|c|c|}
\hline \multicolumn{4}{|c|}{ ¿Adapta El Contenido De Sus Informaciones A Las Distintas Plataformas De Difusión? } \\
\hline \multirow[t]{2}{*}{ SI } & Web Y Rrss & Solo Web & Sólo Rrss \\
\hline & \begin{tabular}{|ll} 
- & Celia Bermejo, Canal \\
& Málaga RTV -Radio \\
- & Manuel Álvarez, Onda \\
& Cero Málaga \\
- & Jesús Sánchez, Cadena \\
& SER Málaga
\end{tabular} & \begin{tabular}{ll|}
$\cdot$ & Romina Zace, \\
& 101 TV \\
Marta \\
Jiménez, Viva \\
Málaga/On- \\
daluz TV
\end{tabular} & $\begin{array}{l}\text { - Ángel Recio, } \\
\text { Málaga Hoy }\end{array}$ \\
\hline $\mathrm{NO}$ & \multicolumn{3}{|c|}{ - Juan Miguel Domínguez, PTVTELECOM } \\
\hline $\begin{array}{l}\text { Lo hace la persona } \\
\text { o el departamento } \\
\text { responsable }\end{array}$ & \multicolumn{3}{|c|}{$\begin{array}{ll} & \text { Andrés García, Cadena Cope Málaga } \\
\text { - } & \text { Matías Stuber, La Opinión de Málaga } \\
\text { - } & \text { Manuel García, Canal Málaga RTV -Televisió- } \\
\text { Pilar R. Quirón, Diario SUR }\end{array}$} \\
\hline
\end{tabular}

Fuente: elaboración propia a partir de los datos obtenidos a través de las entrevistas en profundidad

En cuanto a las ventajas e inconvenientesque el periodista actual encuentra con el uso del Smartphone en su trabajo (véase tabla 2), llama la atención el grado de coincidencia en las respuestas y el hecho de que una misma palabra haya sido utilizada para expresar tanto la principal ventaja, así como el principal inconveniente que ha traído consigo el teléfono inteligente al contexto periodístico. La "inmediatez" es el término al que se alude en ambos casos. Como ventaja se relaciona también con palabras como "rapidez" o "actualidad". En el caso opuesto, la exigencia de "inmediatez" a la profesión se relaciona con consecuencias negativas a través de vocablos o expresiones como "desprofesionalización", "hombre orquesta", "guerra entre periodistas", "reducción de costes", "defectos de forma y contenido", "errores". 
Figura 1. Nube de etiquetas entorno a las ventajas e inconvenientes

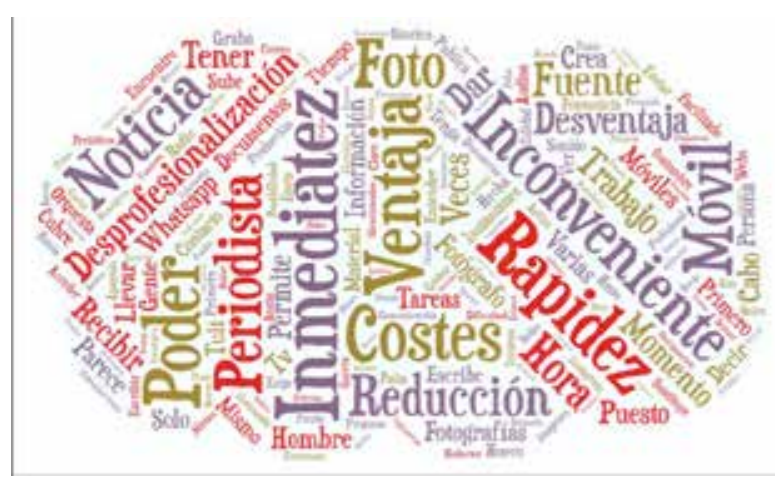

Fuente: elaboración propia a partir de los datos obtenidos a través de las entrevistas en profundidad

Veamos con más detenimiento este capítulo sobre los perjuicios que considera el sector respecto al uso del Smartphone en sus rutinas, a través de algunos extractos de las entrevistas realizadas. "El inconveniente principal para mí es la 'desprofesionalización' del material que se crea. Es decir, entender que una foto hecha con el móvil sustituye a la de un fotógrafo profesional o que se tenga por costumbre que un solo periodista cubre una noticia con un móvil" (Celia Bermejo Pérez, Canal Málaga RTV -Radio-). "Cualquier persona puede ser periodista” (Romina Zace, $101 \mathrm{TV})$. "El inconveniente es que te hace ser hombre orquesta, estar con todo a la vez y tener que hacer fotografías ante la falta de personal contratado, y, lógicamente, se pierde calidad” (Ángel Recio, Málaga Hoy). "Los móviles han provocado una guerra entre periodistas a la hora de ver quién es el primero en dar la noticia, con el riesgo que conlleva" (Manuel García, Canal Málaga RTV -Televisión-). "Se exige un trabajo periodístico inmediato por la simple razón de que es posible y eso no tiene por qué ser periodismo. La reducción de costes está haciendo que las condiciones laborales de los periodistas sean peligrosas para su pervivencia y para la sociedad en general” (Manuel Álvarez, Onda Cero Radio). "Esta rapidez y ansias por ser el primero puede derivar en incurrir en defectos de forma y contenido, o peor aún, provocar una noticia falsa por no corroborar la veracidad de la fuente" (Juan Miguel Domínguez, PTVTELECOM). "A veces las prisas provocan demasiados errores” (Jesús Sánchez, Cadena SER Málaga).

Por último, respecto a la categoría Smartphone como fuente de información, los profesionales aluden a la agilidad y la diversidad que ha aportado el nuevo dispositivo para una comunicación directa con las fuentes de información. "Es una forma de estar en contacto con muchísima gente que conozco o que no y que en el mundo offline sería imposible de gestionar" (Celia Bermejo Pérez, Canal Málaga RTV -Radio $\square$ ). Sin embargo, preguntados sobre en qué medida se nutren de informaciones generadas por ciudadanos particulares o colectivos sociales que llegan a su dispositivo móvil, los periodistas se muestran reticentes, apuntando hacia la necesidad de contrastar la veracidad de la información recibida y de la fuente. "No utilizo ningún material que haya podido ser elaborado por algún particular. Si es un colectivo, depende si es relevante y se le aplica el correspondiente trato periodístico" (Matías Stuber, La Opinión de Málaga). "Vemos cosas a través de Twitter, pero con mucha prudencia porque siempre hay que comprobarlo todo, máxime ahora que en las redes sociales se publican muchas mentiras o medias verdades" (Ángel Recio, Málaga Hoy). "Es poco fiable. Me puede poner en la pista, pero no es suficiente. Tengo que contrastar todas las noticias" (Jesús Sánchez, Cadena SER Málaga). "Poco caso le hago a lo que publican los ciudadanos en redes sociales a la hora de elaborar las noticias. Salvo que sea una alarma generalizada que ya 
sirve como punto de partida a la hora de iniciar el trabajo” (Manuel García, Canal Málaga RTV -Televisión-).

\section{DISCUSIÓN Y CONCLUSIONES}

Las empresas mediáticas son conscientes de la importancia actual de estar presentes en el entorno móvil, conocedoras del creciente consumo de información a través de smartphones. Una amplia mayoría de los medios locales examinados cuenta con una aplicación específica para dispositivos móviles que se actualiza constantemente. En menos de la mitad de los casos analizados, las compañías de comunicación cuentan con personal que se dedique específicamente a dar forma y publicar los contenidos en las distintas plataformas de difusión digital; un hecho que no debemos sobredimensionar teniendo en cuenta que se trata de medios locales, donde los presupuestos no posibilitan, en muchas ocasiones, este tipo de nivel de especialización y donde, generalmente, son los propios periodistas (bajo el concepto de "hombre/mujer orquesta") los que llevan a cabo estas funciones. En este sentido, esta investigación se plantea como punto de partida para un abordaje mayor sobre el examen de cada una de las cuestiones que en ella se proponen (el Smartphone como dispositivo multifunción, metamedio y fuente de información ciudadana), es decir, este estudio abre la posibilidad, dado que bajo nuestra consideración es posible extrapolar el análisis de contenido realizado a un nivel territorial más amplio (véase autonómico o nacional), de ampliar la muestra a un contexto geográfico distinto del planteado en este trabajo, así como a un número mayor de empresas mediáticas y profesionales. Futuras investigaciones pueden contribuir a contrastar y dar validez a los resultados que se exponen, en el objetivo de profundizar en cómo las nuevas tecnologías en general -y el Smartphone en particular- están transformando la profesión periodística.

La asignatura pendiente de las empresas mediáticas malagueñas sería la formación de sus periodistas, y es que muchos de ellos, los denominados "nativos digitales", quizás no la precisen, pero las generaciones predecesoras, aunque adaptadas a los nuevos dispositivos, requieren en ocasiones de un apoyo adicional para sacar a los teléfonos inteligentes el máximo rendimiento en sus rutinas diarias.

El Smartphone ha transformado la profesión del periodista, facilitando su trabajo diario al poner al alcance de su mano nuevas herramientas (cámara, bloc de notas, grabadora) que ahora confluyen en un pequeño dispositivo. Los teléfonos inteligentes también han dotado al sector de una mayor facilidad de acceso a la información y de una inmediatez impensable hace sólo algunos años. Esta inmediatez es, al mismo tiempo, la principal ventaja y el principal inconveniente que los periodistas encuentran en el uso de los smartphones en su profesión. La inmediatez de la que los redactores disponen a la hora de acceder a la información es la misma que a ellos se les exige al redactarla y publicarla. Esto genera, en muchas ocasiones, una lucha entre los propios profesionales por ser los primeros en difundir la noticia, lo que conlleva que, ocasionalmente se produzcan defectos de forma y contenido, errores e incluso noticias falsas o no contrastadas.

Los teléfonos inteligentes han abierto la puerta a nuevas funciones del profesional de la información que, hoy en día, al menos en el ámbito local, rara vez se dedica en exclusiva a la redacción de la pieza noticiosa. Labores como la fotografía, la grabación y edición de videos o la gestión de web y redes sociales, todo ello posible a través 
del Smartphone, son funciones añadidas a la profesión. Es lo que González (2013, p. 10) denomina "periodista orquesta". La polivalencia es, por tanto, una de las características predominantes entre los profesionales de las empresas mediáticas locales. Siguiendo a Micó (2006) está polivalencia se daría en los tres tipos que distingue el autor: mediático, tecnológico y temático. Ahora bien, los profesionales del sector apuntan a la desprofesionalización y a la disminución de costes por unificación de tareas en una sola persona con la consiguiente reducción de personal, como consecuencias negativas de las nuevas funciones que posibilitan los smartphones. Pese a realizar multitud de trabajos a través del dispositivo móvil, el redactor sigue empleando su PC para maquetar la información, por lo que, en términos generales, los profesionales aún no se identifican con el término "Mobile Journalist".

El periodista actual utiliza su teléfono inteligente para mantenerse informado de las últimas noticias, empleando para ello, de forma mayoritaria, Twitter o las webs y/o aplicaciones de los principales medios de comunicación -especialmente prensa $\square$ locales y nacionales, por lo que el Smartphone se convierte en "metamedio" (Márquez,2017) contenedor de muchos otros medios viejos y nuevos.

El sector periodístico valora la agilidad que los teléfonos inteligentes aportan a la comunicación con sus fuentes, principalmente a través de WhatsApp, pero se muestra receloso a la hora de incorporar a las noticias las informaciones procedentes de ciudadanos particulares o colectivos sociales de los que se nutre. Cuando hace esto último, será únicamente y por el momento, para seguir la pista de acontecimientos relevantes. Previsiblemente, y de forma paulatina, los medios de comunicación y la profesión periodística en particular, irán incorporando medios que faciliten la participación directa de la ciudadanía en el intento de proveerse de los, cada vez más necesarios, espacios de comunicación y socialización con las audiencias (Lara, 2008).

\section{NOTAS}

1 Véase: http://www.juntadeandalucia.es/agenda/pages/detalleOrganismo.and?parametro=330

2 Aunque aparece así en el registro del organismo autonómico, la denominación de la empresa es Canal Málaga RTV (Empresa Municipal de Gestión de Medios de Comunicación) desde el 1 de enero de 2018.

3 Aunque aparece así en el registro del organismo autonómico, la denominación de la empresa es Canal Málaga RTV (Empresa Municipal de Gestión de Medios de Comunicación) desde el 1 de enero de 2018.

4 En el registro oficial de la Junta de Andalucía aparece como Radio Málaga.

\section{REFERENCIAS}

Aguado, J. M. y Martínez, I. J. (2008). Sociedad Móvil: tecnología, identidad y Cultura. Madrid: Biblioteca Nueva. Ahonen, T. T. (2008). Mobile as 7th of the Mass Media: Cellphone, cameraphone, iPhone, smartphone. London: 
Futuretext.

Bustamante, E. (2011). España y Latinoamérica. Economía creativa del entretenimiento digital. En E. Bustamante (Ed.), Industrias creativas. Amenazas sobre la cultura digital (pp.117-144). Barcelona: Gedisa.

Canavilhas, J. (2013). Modelos informativos para aparatos móviles: información hipermultimediática y personalizada. En C. Raymond (Ed.), Hacia el Periodismo Móvil (pp.20-32). Santiago de Chile: Revista Mediterránea de Comunicación, Colección Mundo Digital / 3. Recuperado de https://goo.gl/C8FeqU

Casero-Ripollés, A. (2010). Prensa en internet: nuevos modelos de negocio en el escenario de la convergencia. En El profesional de la Información, 19 (6), 595-601. doi: 10.3145/epi.2010.nov05. Recuperado de https://goo.gl/ h5QnuK

Costa, C. (2012). Ciberperiodismo en el Smartphone. Estudio de la multimedialidad, usabilidad, hipertextualidad e interactividad de las aplicaciones de medios nativos digitales para Smartphone. En Estudios sobre el Mensaje Periodístico, 18, núm. especial octubre, 243-251. Recuperado de https://goo.gl/cZxhWE

García-Avilés, J.; Meier, K.; Kaltenbrunner, A.; Carvajal, M. y Kraus, D. (2009). Newsroom integration in Austria, Spain and Germany: Models of Media Convergence. En JournalismPractice, 3 (3), 285-303. Recuperado de https:// goo.gl/S2vVN2

Gardner, H. Y Davis, K. (2014). La generación APP. Barcelona: Paidós.

González-Molina, S (2013). La polivalencia profesional de los periodistas en el entorno móvil. En C. Raymond (Ed.), Hacia el Periodismo Móvil (pp.7-19). Santiago de Chile: Revista Mediterránea de Comunicación, Colección Mundo Digital / 3. Recuperado de https://goo.gl/WQJujy

Huang, E.; Rademakers, L.; Fayemiwo, M.A. y Dunlap, L. (2004).Converged Journalism and Quality: A Case Study on The Tampa Tribune News Stories. EnConvergence, 10 (4), 73-91.

Jenkins, H. (2006). Convergence Culture. Where old and new media collide. Nueva York: New York Press.

Kerckhove, D. (2005). Los sesgos de la electricidad (Lección inaugural del curso académico 2005-2006 de la UOC). FUOC, Fundación para la Universitat Oberta de Catalunya. Recuperado de https://goo.gl/AjGQ7Y

Lara, T. (2008). La nueva esfera pública. Los medios de comunicación como redes sociales. En Telos:Cuadernos de Comunicación e Innovación, 76. Recuperado dehttps://goo.gl/bmuw4n

López-García, X.; Westlund, O. y Silva Rodríguez, A. (2015). Prensa, smartphones y tablets. La industria de medios impresos se sube al periodismo móvil. En Telos: Cuadernos de Comunicación e Innovación, 100, 128-139. Recuperado de https://goo.gl/N4Rpo1

Márquez, I. (2017). El Smartphone como metamedio. En Observatorio Journal, 061-071. Recuperado de https:// goo.gl/mWY8pW

Marta Lazo, C. y García Idiakez, M. (2014). El uso profesional de la red social Twitter en la redacción del diario español El País. En Palabra Clave, 17(2), 353-377. Recuperado de https://goo.gl/hq1nhw

Melle-Goyanes, M. y López-García, X. (2017). Narrativas Transmedia y Periodismo Móvil: expansión de un suceso 
noticiable a través de múltiples plataformas. En A. De Lara, y F. Arias, Metamorfosis. Perspectivas sobre la innovación en periodismo (pp. 145-163), Libro de actas del XXXIII Congreso Internacional de la Sociedad Española Periodística, Alicante: Universidad Miguel Hernández de Elche.

Micó, J.L. (2011). Periodistas audiovisuales inmóviles ante el periodismo multimedia móvil. En A. Casero Ripollés y J. Marzal (eds.). Periodismo en televisión. Nuevos horizontes, nuevas tendencias. Sevilla, Salamanca, Zamora: Comunicación Social.

Micó, J.L. (2006). Periodisme a la xarxa: Llenguatgenoud'unoficivell. Vic: Eumo.

Miller, J. (2014). The fourth screen: Mediatization and the Smartphone. En Mobile Media \&Communication, 2 (2), 209-226. doi: 10.1177/2050157914521412. Recuperado de https://goo.gl/PZ9ksg

Quinn, S. (2009). MoJo. Mobile Journalism in the Asian Region. Singapore: Konrad-Adenauer- Stiftung.

Quiróz Y. R. (2016). El smartphone como herramienta del periodismo digital. En Cultura: Lima (Perú), 30, 109-139. Reigosa, C. G. (2007). La actualidad contra el periodismo. En Cuadernos de periodistas, 9, 19-28. Recuperado de https://goo.gl/nrhDEY

Said Hung, E., Serrano Tellería, A., García de Torres, E., Calderín, M., Rost, A., Arcila Calderón, C., ...Sánchez Badillo, J. (2013). Ibero American Online News Managers' Goals and Handicaps in Managing Social Media. En Television and New Media. doi: 10.1177/1527476412474352

Sainz de Baranda, C.; Limón, N. y Herrero, E. (2012). ¿Connectingpeople? el uso periodístico de los smartphones (pp. 59-69), Congreso Internacional Periodismo en Red (edición). CD ROM. ISBN: 978-84-615-6211-4: (V). Copyrigt(C) 2012congresoperiodismoenred.es. Recuperado de https://goo.gl/xHtrkm

Sánchez-González, M. (2011). Los smartphones como herramienta para el periodismo móvil: potencial y tendencias de uso por profesionales de la información, usuarios y empresas periodísticas. En J. M. de Pablos Coello (Presidencia), Periodismo y tecnologías en la producción, distribución y recepción. Simposio llevado a cabo en el III Congreso Internacional Latina de Comunicación Social, Universidad de La Laguna, Tenerife. Recuperado de https://goo. $\mathrm{gl} / 3 \mathrm{wds} 82$

Varona, D.; Sánchez, M.; Arrocha, R. (2017). Consumo de información política en dispositivos móviles en España: caracterización del usuario tipo y su interacción con las noticias. En El profesional de la información, 26 (4), 641 648. doi: 10.3145/epi.2017.jul.08. Recuperado de https://goo.gl/8CeMFE

Westlund, O. (2013). Mobile news. A review and model of journalism in an age of mobile media. En Digital Journalism, 1 (1), 6-26. doi: 10.1080/21670811.2012.740273. Recuperado de https://goo.gl/Hz56C5

Westlund, O. (2015). News Consumption in an Age of Mobile Media: Patterns, People, Place and Participation. EnMobile Media \& Communication, 3 (2). doi: 10.1177/2050157914563369

Williams, B. A. y Delli-Carpini, M. (2011). After Broadcast News. Media Regimes, Democracy, and the New Information Environment. Nueva York: Cambridge University Press. 